\title{
Fuzzy Inference Systems Type-1 and Type-2 for Digital Images Edge Detection
}

\author{
Olivia Mendoza, Patricia Melín, Guillermo Licea Sandoval
}

\begin{abstract}
Edges detection in digital images is a problem that has been solved by means of the application of different techniques from digital signal processing, also the combination of some of these techniques with Fuzzy Inference System (FIS) has been experienced. In this work a new FIS Type-2 method is implemented for the detection of edges and the results of three different techniques for the same intention are compared.
\end{abstract}

Index Terms-Fuzzy Logic, Type-2 FIS, Sobel Operator, Border Detection.

\section{INTRODUCTION}

In the area of digital signal processing, methods have been proven that solve the problem of image recognition. Some of them include techniques like binarization, bidimensional filtrate, detection of edges and compression using banks of filters and trees [ 1 ], among others.

Specifically in methods for the detection of edges we can find comparative studies of methods like: Canny, Narwa, Iverson, Bergholm y Rothwell [2]. Others methods can group in two categories: Gradient and Laplacian.

The gradient methods like Roberts, Prewitt and Sobel detect edges, looking for maximum and minimum in first derived from the image. The Laplacian methods like Marrs-Hildreth do it finding the zeros of second derived from the image [3].

This work is the beginning of an effort for the design of new pre-processing images techniques, using Fuzzy Inference Systems (FIS), that allow feature extraction and construction of input vectors for neural networks with aims of image recognition.

Artificial neural networks are one of the most used objective techniques in the automatic recognition of patterns, here some reasons:

- Theoretically any function can be determined.

- Except the input patterns, it is not necessary to provide additional information.

- They are possible to be applied to any type of patterns and to any data type. [4]

Manuscript submitted November 6, 2006.

O. Mendoza, is student of Doctorate in the Investigation and Post-degree Department of Universidad Autonoma de Baja California, Tijuana, Mexico, (Tel.: +1 52664682 1033; fax: +1 52664682 2700. e-mail address: omendoza@uabc.mx).

P. Melin is with Department of Computer Science, Tijuana Institute of Technology, Tijuana, Mexico, (Tel.: +1 52664623 6318; fax: +1 52664623 6318. e-mail address: pmelin@tectijuana.mx).
The idea to apply artificial neuronal networks for images recognition, tries to obtain results without providing another data that the original images, of this form the process is more similar to the form in which the biological brain learns to recognize patterns, only knowing experiences of past.

Models with modular neural networks have been designed, that allow to recognize images divided in four or six parts, which is necessary by the great amount of input data, since an image without processing of $100 \times 100$ pixels, needs a vector 10000 elements, where each one corresponds to pixel with variations of gray tones between 0 and 255 [5].

This work shows an efficient Fuzzy Inference System for edges detection, in order to use the output image like input data for modular neural networks.

In the propose technique, it is necessary to apply Sobel operators to the original images, then use a Fuzzy Inference System to generate the vector of edges that would serve like input data in a neural network.

\section{SOBEl Operator}

Sobel operator applied on a digital image in gray scale, calculates the gradient of the intensity of brightness of each pixel, giving the direction of the greater possible increase of black to white, in addition calculates the amount of change of that direction.

\section{A. Calculation of the gradient and magnitude of the gradient}

The Sobel operator performs a 2-D spatial gradient measurement on an image. Typically it is used to find the approximate absolute gradient magnitude at each point in an input grayscale image.

The Sobel edges detector uses a pair of $3 \times 3$ convolution masks, one estimating the gradient in the $\mathrm{x}$-direction (columns) and the other estimating the gradient in the $y$ direction (rows).

A convolution mask is usually much smaller than the actual image. As a result, the mask is slid over the image, manipulating a square of pixels at a time. The Sobel masks are shown in (1) [6]:

$$
\text { Sobel }_{x}=\left[\begin{array}{lll}
-1 & 0 & 1 \\
-2 & 0 & 2 \\
-1 & 0 & 1
\end{array}\right] \quad \text { Sobel }_{y}=\left[\begin{array}{ccc}
1 & 2 & 1 \\
0 & 0 & 0 \\
-1 & -2 & -1
\end{array}\right]
$$


Where Sobely y Sobelx are the Sobel Operators throughout $\mathrm{x}$-axis and $\mathrm{y}$-axis.

If we define $I$ as the source image, $g_{x}$ and $g_{\mathrm{y}}$ are two images which at each point contain the horizontal and vertical derivative approximations, the latter are computed as (2) and (3).

$$
\begin{aligned}
& g_{x}=\sum_{i=1}^{i=3} \sum_{j=1}^{j=3} \text { Sobel }_{x, i, j} * I_{r+i-2, c+j-2} \\
& g_{y}=\sum_{i=1}^{i=3} \sum_{j=1}^{j=3} \text { Sobel }_{y, i, j} * I_{r+i-2, c+j-2}
\end{aligned}
$$

Where $g x$ and $g y$ are the gradients along axis-x and axis-y, and * represents the convolution operator.

The gradient magnitude $g$ calculates with (4) [7].

$$
g=\sqrt{g_{x}^{2}+g_{y}^{2}}
$$

\section{Edges Detection By Gradient Magnitude}

Although the intention of this work, is to verify the efficiency of a FIS for edges detection in digital images, from the approaches given by Sobel operator, is necessary to display first the obtained results using only the gradient magnitude.

It will be used as example the first image of the subject number one of the ORL (figure 1) [8]. The gray tone of each pixel of this image is a value of between 0 and 255 .

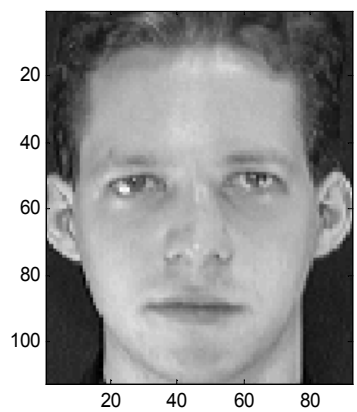

Fig. 1. Original Image 1.pgm

In figure 2 appears the image generated by $g x$, and figure 3 presents the image generated by $g y$.

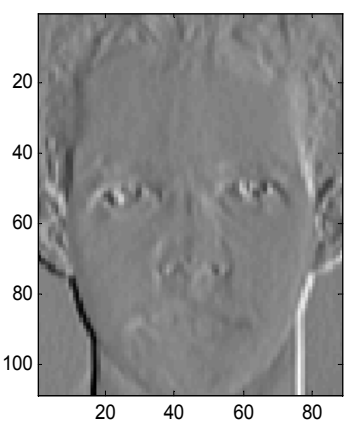

Fig. 2 Image given by $g x$

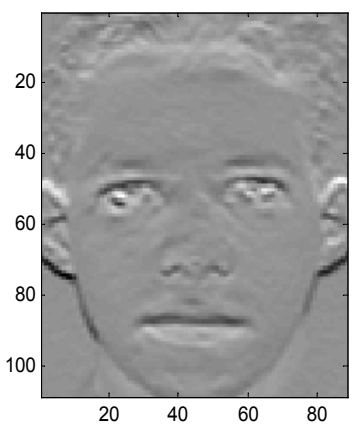

Fig. 3 Image given by gy
An example of maximum and minimum values of the matrix given by $g x$, gy and $g$ from the image 1.pgm showns in table 1.

TABLE 1. MAXIMUM AND MINIMUM VALUES FROM 1.PGM, GX, GY Y G.

\begin{tabular}{|l|l|l|l|l|}
\hline Tone & $1 . p g m$ & gx & gy & g \\
\hline Minimum & 11 & -725 & -778 & 0 \\
\hline Maximum & 234 & 738 & 494 & 792 \\
\hline
\end{tabular}

After applying (4), $\mathrm{g}$ is obtained as it is in figure 4.

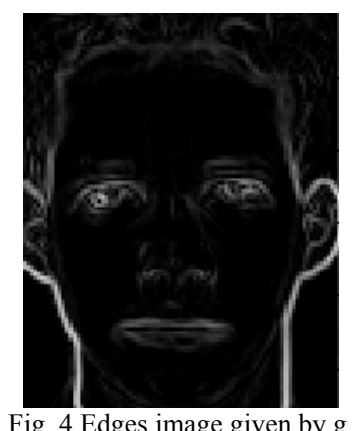

\section{Edges Detection by FIS TYPE 1}

A Mamdani FIS was implemented using Type-1 Fuzzy Logic, with four inputs, one output and 7 rules, using the Matlab Fuzzy Logic Tool Box 7 [9], like is in figure 5.

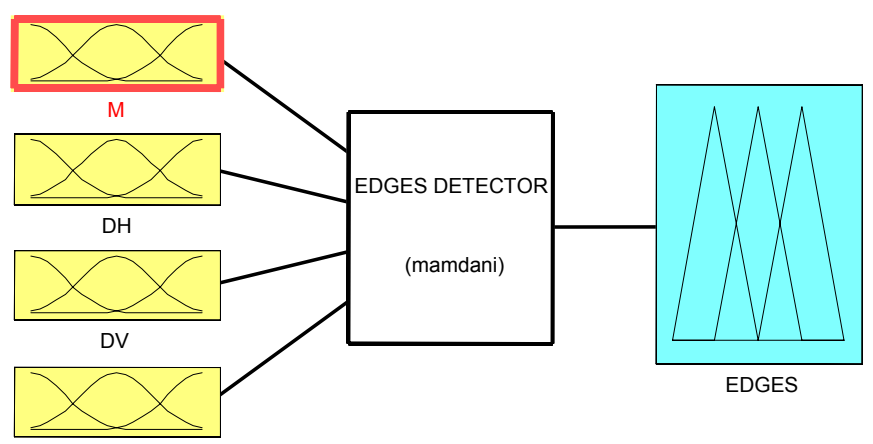

Fig. 5 FIS in Matlab Fuzzy Logic Tool Box. 


\section{A. Inputs for FIS Type 1}

For the Fuzzy Inference System Type-1, 4 inputs are required, 2 of them are the gradients with respect to $\mathrm{x}$-axis and y-axis, calculated with (2) and (3), to which we will call DH and DV respectively.

The other two inputs are filters that calculate when applying two masks by convolution to the original image. A high-pass filter, given by the mask of the equation (5), and low-pass filter given by the mask of the equation (6).

The high-pass filter hHP detects the contrast of the image to guarantee the border detection in relative low contrast regions. The low-pass filter hMF allow to detects image pixels belonging to regions of the input were the mean gray level is lower. These regions are proportionally more affected by noise, supposed it is uniformly distributed over the whole image.

The goal here is to design a system which makes it easier to include edges in low contrast regions, but which does not favour false edges by effect of noise. [10]

$$
\begin{aligned}
h H P & =\left[\begin{array}{rrr}
-\frac{1}{16} & -\frac{1}{8} & -\frac{1}{16} \\
-\frac{1}{8} & \frac{3}{4} & -\frac{1}{8} \\
-\frac{1}{16} & -\frac{1}{8} & -\frac{1}{16}
\end{array}\right] \\
h M F & =\frac{1}{25} *\left[\begin{array}{lllll}
1 & 1 & 1 & 1 & 1 \\
1 & 1 & 1 & 1 & 1 \\
1 & 1 & 1 & 1 & 1 \\
1 & 1 & 1 & 1 & 1 \\
1 & 1 & 1 & 1 & 1
\end{array}\right]
\end{aligned}
$$

Then the inputs for FIS type 1 are:

$$
\begin{aligned}
& \mathrm{DH}=g_{x} \\
& \mathrm{DV}=g_{y} \\
& \mathrm{HP}=h H P^{* I} \\
& \mathrm{M}=h M F^{*} I
\end{aligned}
$$

where $*$ is the convolution operator.

\section{B. Fuzzy variables}

For all the fuzzy variables, the membership functions are Gaussian.

According to the executed tests, the values in DH and DV, go from -800 to 800 , then the ranks in X-axis adjusted as it is in figures 6,7 and 8 , in where the membership functions are:

LOW: gaussmf(43,0),

MEDIUM: gaussmf(43,127),

HIGH: gaussmf(43,255).

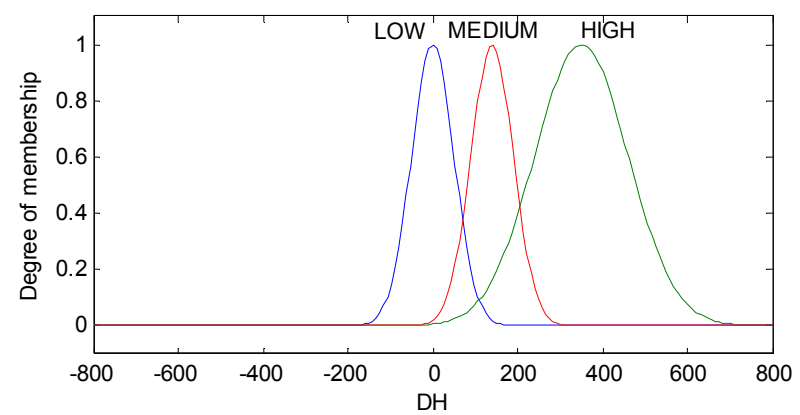

Fig. 6 Input variable DH

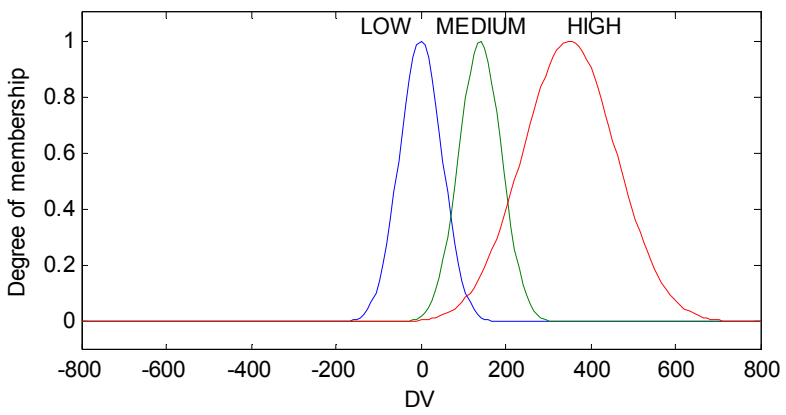

Fig. 7 Input variable DV

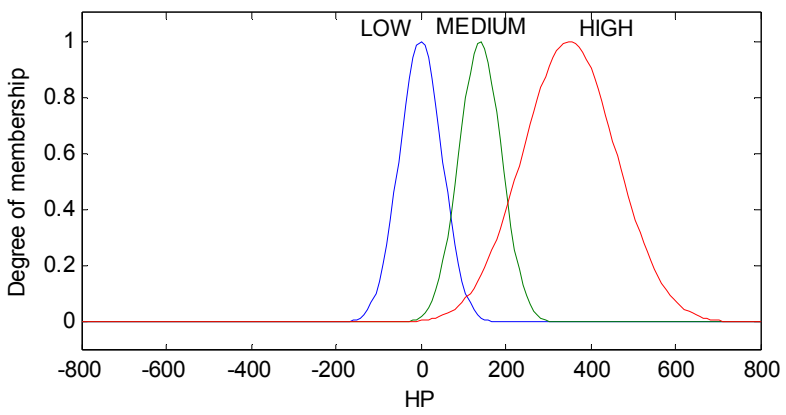

Fig. 8 Input variable HP

In the case of variable $M$, the tests threw values in the rank from 0 to 255, and thus the rank in $\mathrm{x}$-axis adjusted, as it is appraised in figure 9 .

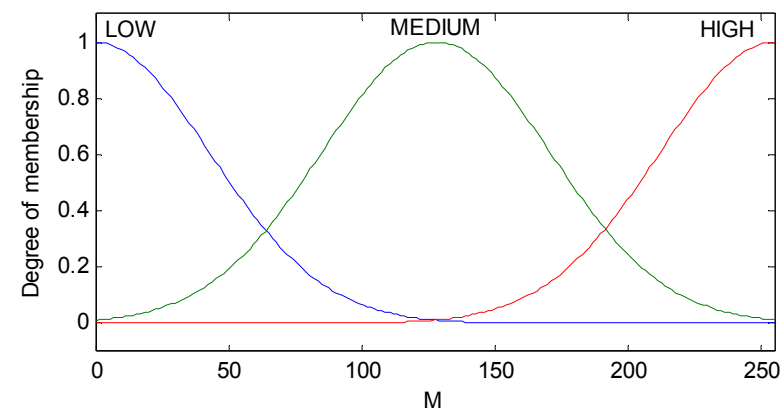

Fig. 9 Input variable $M$ 
In figure 10 is the output variable EDGES that also adjusted the ranks between 0 and 255, since it is the range of values required to display the edges of an image.

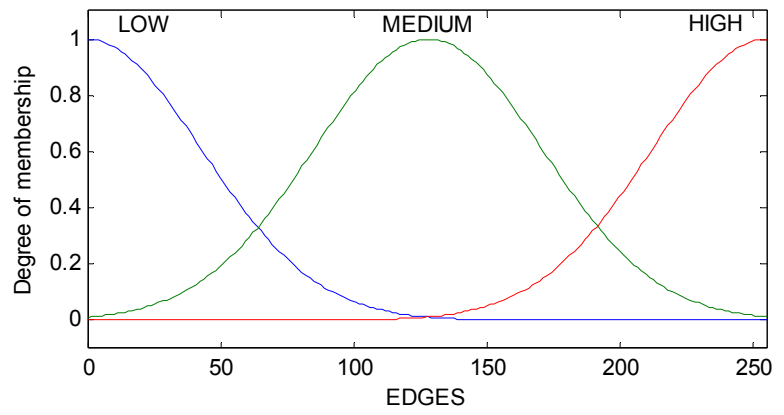

Fig.10 Output variable EDGES

\section{Fuzzy Inference Rules}

The seven fuzzy rules that allow to evaluate the input variables, so that the exit image displays the edges of the image in color near white (HIGH tone), whereas the background was in tones near black (tone LOW).

1. If (DH is LOW) and (DV is LOW) then (EDGES is LOW)

2. If (DH is MEDIUM) and (DV is MEDIUM) then (EDGES is HIGH)

3. If (DH is HIGH) and (DV is HIGH) then (EDGES is HIGH)

4. If (DH is MEDIUM) and (HP is LOW) then (EDGES is HIGH)

5. If (DV is MEDIUM) and (HP is LOW) then (EDGES is HIGH)

6. If ( $\mathrm{M}$ is LOW) and (DV is MEDIUM) then (EDGES is LOW)

7. If (M is LOW) and (DH is MEDIUM) then (EDGES is LOW)

\section{FIS Type 1 Results}

The result obtained for image of figure 1 is remarkably better than the one than it was obtained with the method of gradient magnitude, as it is in figure 11 .

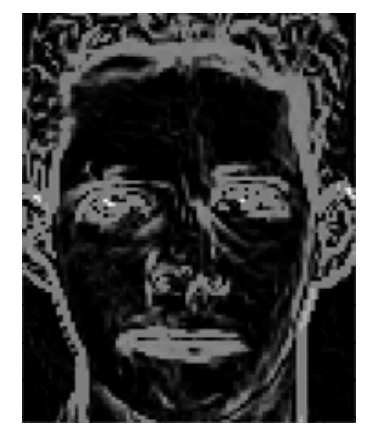

Fig. 11 EDGES Image by FIS Type 1.

Reviewing the values of each pixel, we see that all fall in the rank from 0 to 255, which is not obtained with the method of gradient magnitude.
V. EDGES DETECTION BY A FIS TYPE-2

For FIS Type-2, the same method was followed that in FIS Tupe-1, indeed to be able to make a comparison of both results. The tests with the FIS type-2, were executed using the program imagen_bordes_fis $2 . m$, which creates a Inference System Type-2 (Mamdani) by intervals [11 ] [12 ].

The mentioned program creates the fuzzy variables type 2 as it is seen in figure 12. The wide of the FOU chosen for each membership function was the one that had better results after several experiments.

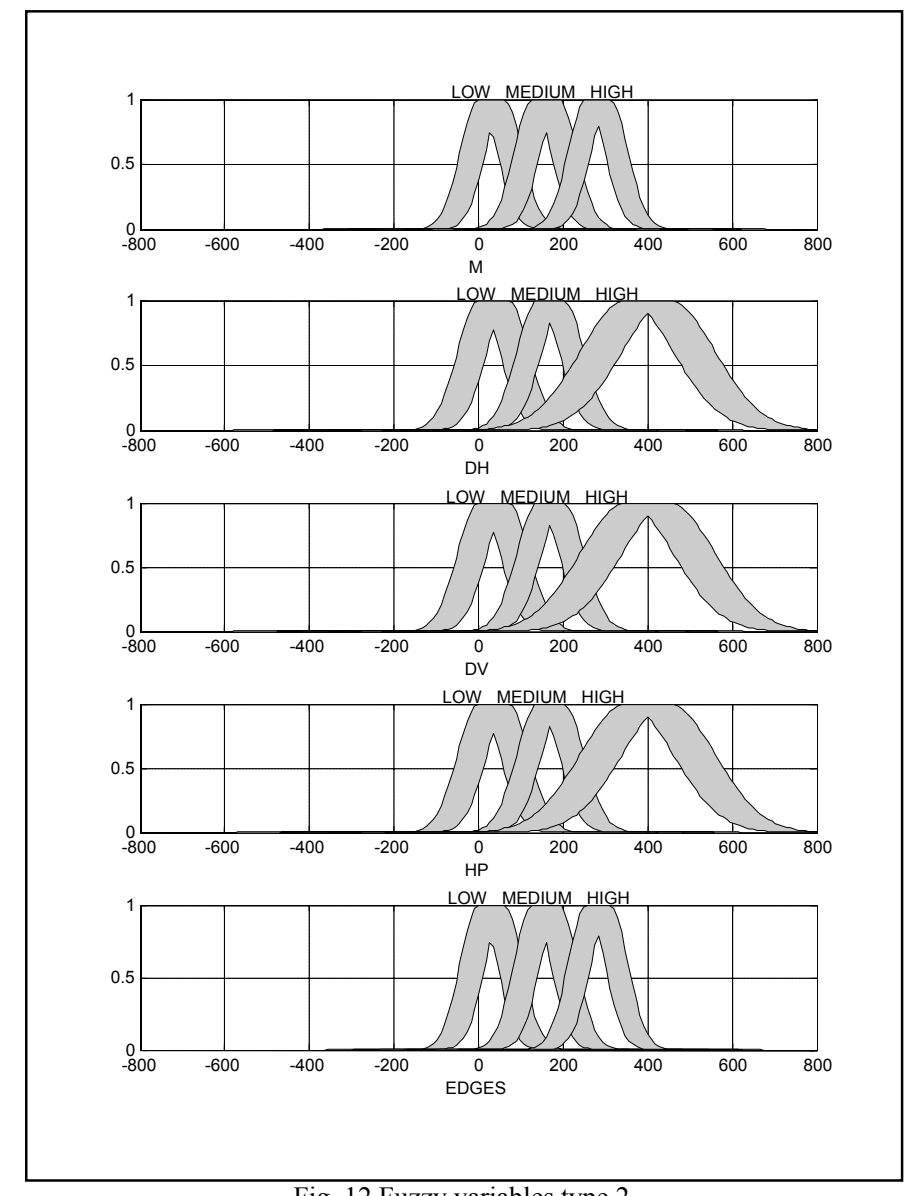

Fig. 12 Fuzzy variables type 2.

The program imagen_bordes_fuzzy2.m was implemented to load the original image, and to apply the filters before mentioned. Because the great amount of data that the diffuse rules must evaluate, the image was divided in four parts, and the FIS Type-2 was applied each one separately.

The result of each evaluation throws a vector with tones of gray by each part of the image, in the end is the complete image with the edges, as it is seen in figure 13. 


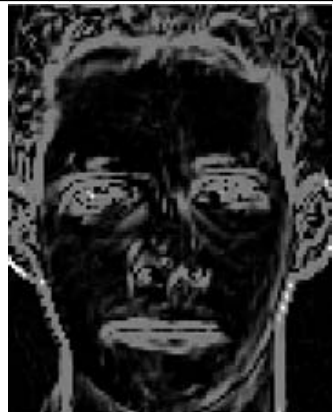

Fig. 13 EDGES Image by FIS Type 2

\section{RESULTS COMPARISON}

The first results of several tests conducted in different images can be appreciated in table 1 .

TABLE 1. Results of EdGE DETECTION By FIS1 Y FIS2 (DARK BACKGROUND)

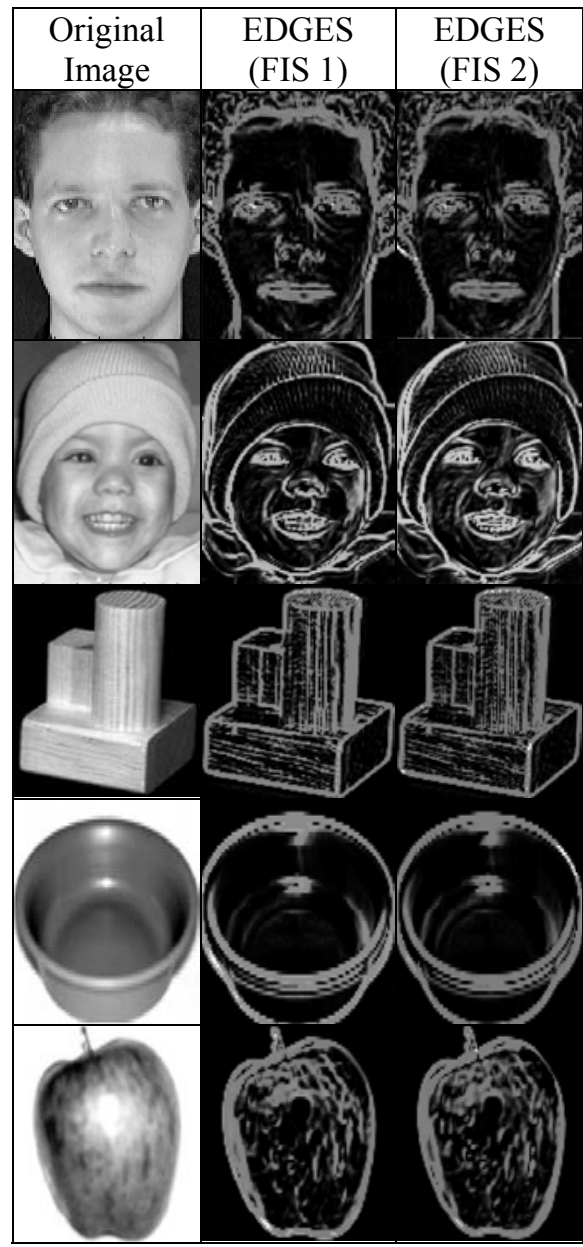

At first the results with FIS Type- 1 and FIS Type 2 are seen very similar. However thinking about that to show the images with a dark background it could confuse the contrast of tones, tests were done investing the consequent of the rules, so that the edges take the dark tone and the bottom the clear tone, the rules changed to the following form:
1. If (DH is LOW) and (DV is LOW) then (EDGES is HIGH)

2. If (DH is MEDIUM) and (DV is MEDIUM) then (EDGES is LOW)

3. If (DH is HIGH) and (DV is HIGH) then (EDGES is LOW)

4. If (DH is MEDIUM) and (HP is LOW) then (EDGES is LOW)

5. If (DV is MEDIUM) and (HP is LOW) then (EDGES is LOW)

6. If (M is LOW) and (DV is MEDIUM) then (EDGES is HIGH)

7. If ( $\mathrm{M}$ is LOW) and (DH is MEDIUM) then (EDGES is HIGH)

Fuzzy Inference Systems was tested both (Type-1 and Type-2), with the new fuzzy rules and same images, obtaining the results that are in table 2 .

TABLE 2. Results OF EDGE DETECTION By FIS1 y FIS2 (CLEAR BACKGROUND)

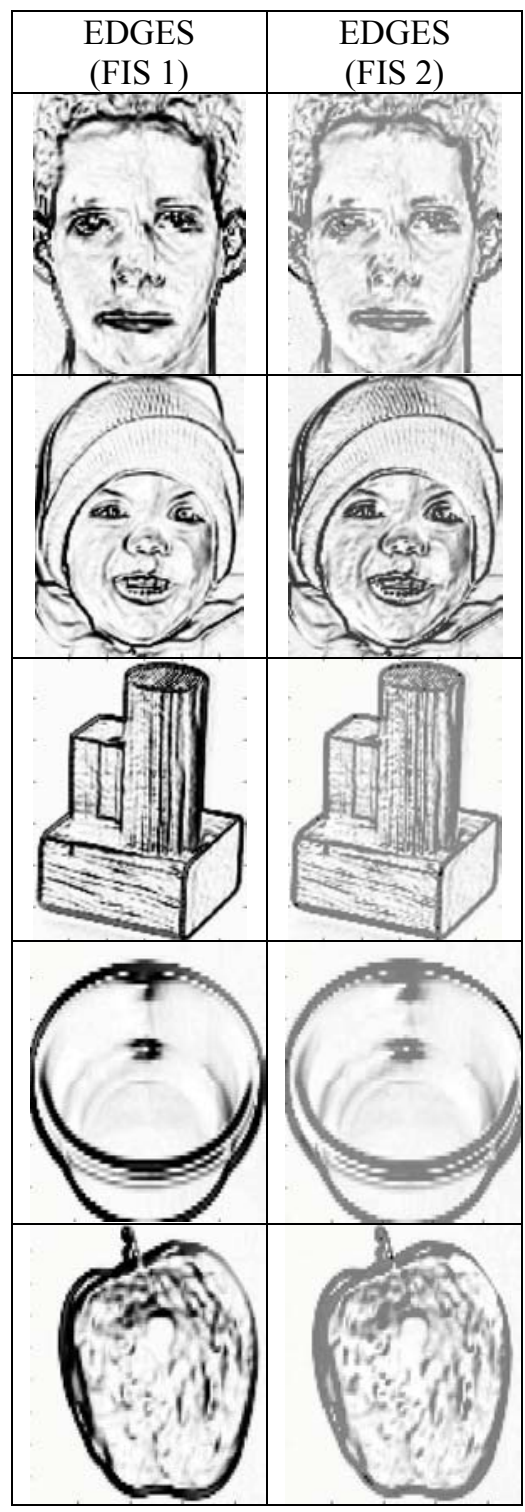

In this second test can be appreciated a great difference between the results obtained with the FIS 1 and FIS 2, noticing at first a greater contrast in the images obtained with the FIS 1 and giving to the impression of a smaller range of tones of gray in the FIS type 2 . 


\section{Engineering Letters, 15:1, EL_15_1 7}

In order to obtain an objective comparison of the images, histograms were elaborated respectively [14] corresponding to the resulting matrices of edges of the FIS 1 and FIS 2, which are in table 3 .

The histograms show in the y-axis the range of tones of gray corresponding to each image and in $\mathrm{x}$-axis the frequency in which he appears pixel with each tone.

TABle 3. Histograms Of The Resulting Images Of The Edges by GRADIENT MAGNITUd, Fis 1 AND FIS 2 METHODS.

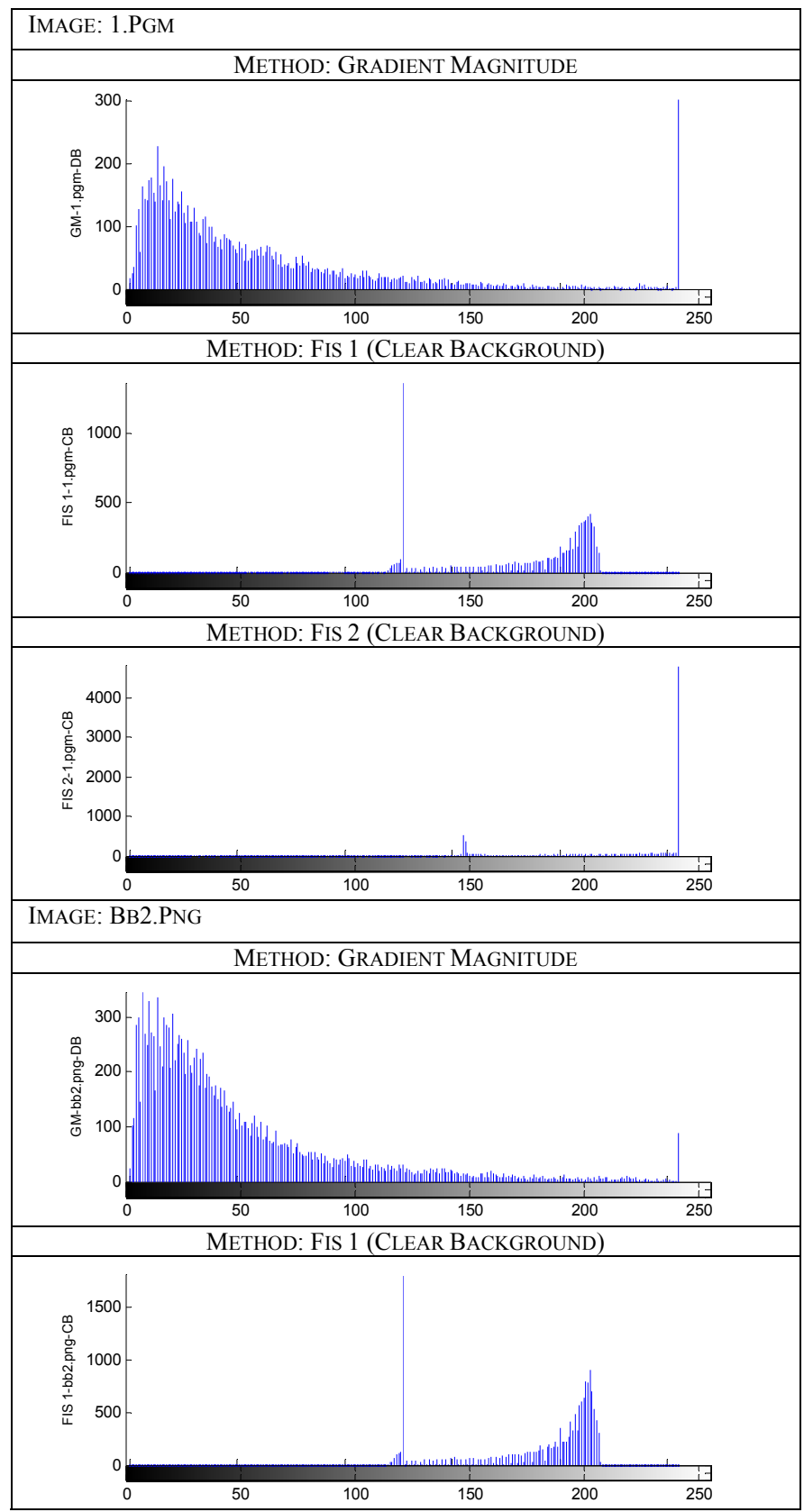

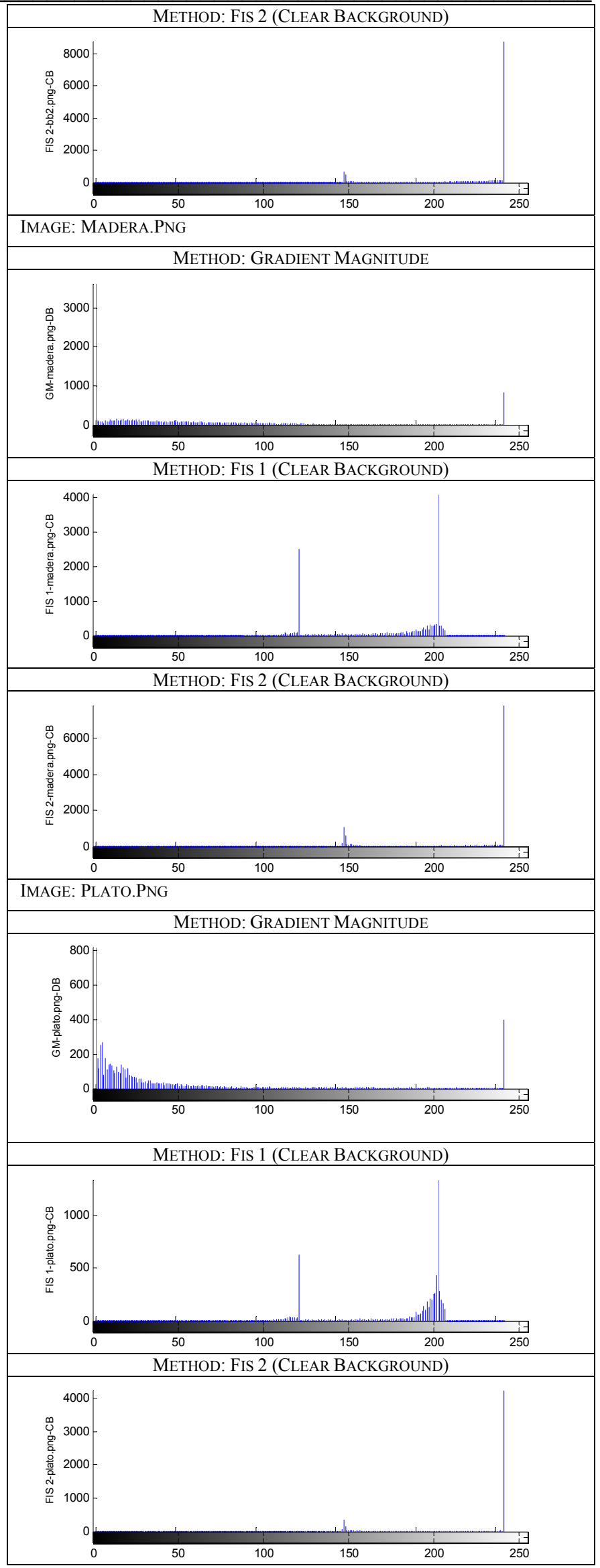


Engineering Letters, 15:1, EL_15_1_7
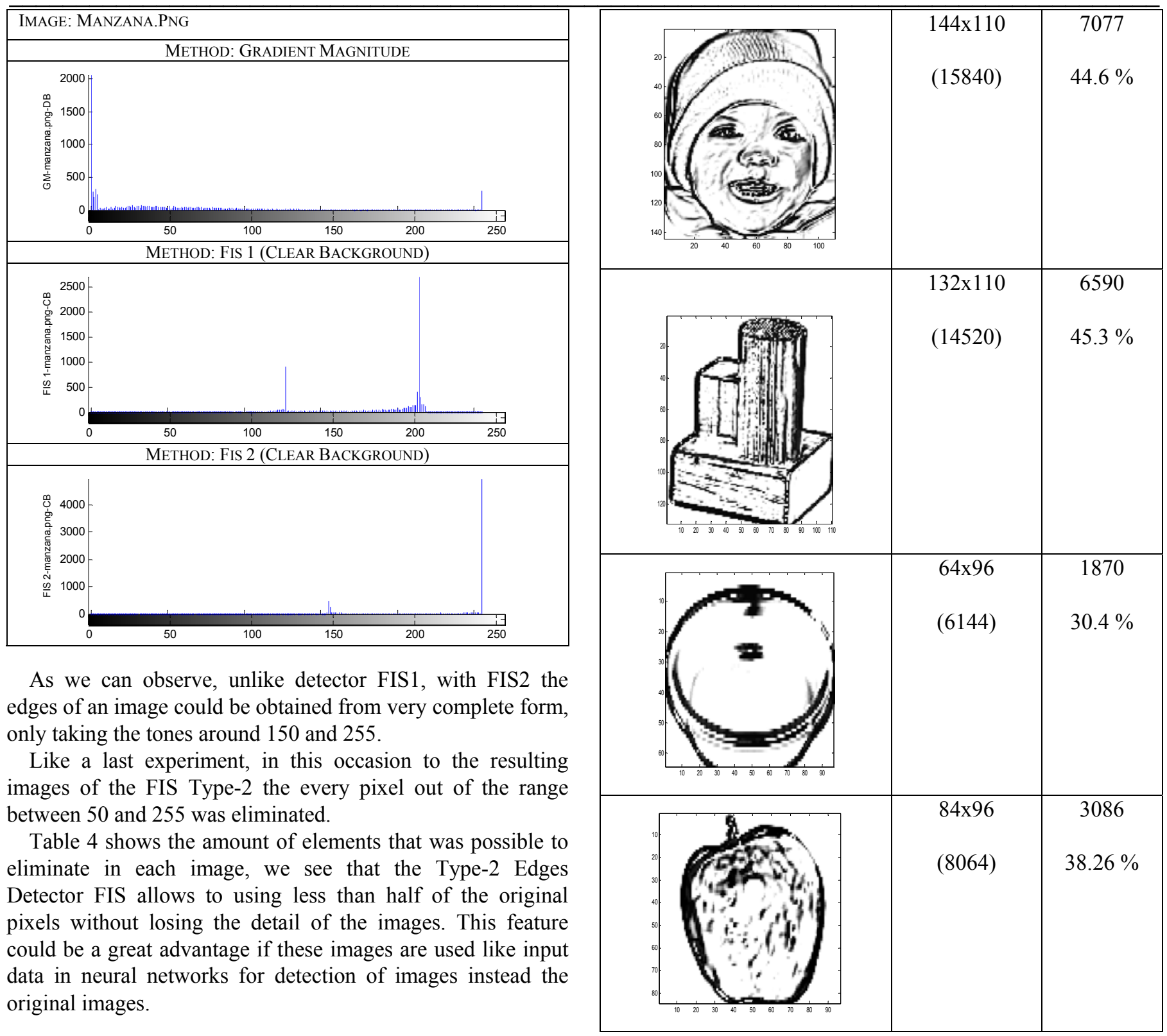

As we can observe, unlike detector FIS1, with FIS2 the edges of an image could be obtained from very complete form, only taking the tones around 150 and 255 .

Like a last experiment, in this occasion to the resulting images of the FIS Type-2 the every pixel out of the range between 50 and 255 was eliminated.

Table 4 shows the amount of elements that was possible to eliminate in each image, we see that the Type-2 Edges Detector FIS allows to using less than half of the original pixels without losing the detail of the images. This feature could be a great advantage if these images are used like input data in neural networks for detection of images instead the original images.

TABLE 4. FIS Type-2 Edges IMAges Including ONLY PIXELS With TONES BETWEEN 150 AND 255

\begin{tabular}{|c|c|c|}
\hline BORDERS IMAGE & $\begin{array}{c}\text { DIMENSION } \\
\text { (pixels) }\end{array}$ & $\begin{array}{c}\text { PIXELS } \\
\text { INCLUDED }\end{array}$ \\
\hline & $108 \times 88$ & 4661 \\
\hline
\end{tabular}

\section{CONCLUSIONS}

The application of Sobel filters was very useful to define the input vectors for the FIS Type-1 and FIS Type-2, although in future works we will try to design Neuro-Fuzzy techniques able to extract image patterns without another data that the original image and to compare the results with traditional techniques of digital signal processing.

Thanks to the histograms of the images it was possible to verify the improvement of results of the FIS Type-1 with respect to the FIS Type-2, since with only the appreciation of the human eye was very difficult to see an objective difference.

The best result was obtained by the Fuzzy Inference System Type-2, because it was possible to clear more than half of the pixels without depreciating the image, which will reduce in drastic form the cost of training in a neuronal network. 


\section{Engineering Letters, 15:1, EL_15_1_7}

An important advantage of the Fuzzy Inference Systems is that diverse variables can be modeled as noise and contrast. This causes that the results are near to the reality and therefore they are more reliable.

\section{REFERENCES}

[1] Luis Enrique Palafox Maestre, (Tijuana México, Mayo del 2002), "Técnicas de Procesamiento de Imágenes Utilizando un Procesador Digital de Señales", Centro de Investigación y Desarrollo de Tecnología Digital,

[2] Michael D. Heath, (Florida, Mayo de 1996), “A Robustal Visual Method for Assessing the Relative Performance of Edge Detection Algorithms", Available: http://marathon.csee.usf.edu/edge/edge_detection.html

[3] Roger Claypoole, Jim Lewis, Srikrishna Bhashyam, Kevin Kelly, (Rice University, Huston, U.S.A.), Curso: "ELEC 539 - Digital Image Processing Rice University", Available: www.owlnet.rice.edu/ elec539/Proyects97/morphjrks/moredge.html

[4] Miguel Angel Martínez Rubio, Mercedes Velásquez Pérez., (Madrid, Abril de 1996), "Técnicas de redes neuronales aplicadas al reconocimiento y clasificación de pixeles de imágenes de satélite", IV Simposio Nacional de Predicción.

[5] Olivia Mendoza Duarte, Patricia Melín, (Tijuana, Mexico, Octubre de 2005), "The Fuzzy Sugeno Integral as a Decision Operator in the Recognition of Images with Modular Neural Networks", International Conference on Fuzzy Systems, Neural Networks and Genetic Algorithms FNG'05,

[6] Hill Green, (Philadelphia, 2002), "Edge Detection Tutorial", Available: http://www.pages.drexel.edu/ weg22/edge.html

[7] Yuan Fan, Lalita Udpa, Pradeep Ramuhalli, William Shih, George C. Stockman, "Automated Rivet Inspection for Aging Aircraft with Magneto-optic Image", International Workshop on Electromagnetic Nondestructive Evaluation, East Lansing, (Michigan, Junio de 2004).

[8] AT\&T Laboratories, Cambridge, 2002, "The ORL Database of Faces", http://www.uk.research.att.com/facedatabase.html, (Mayo de 2004).

[9] The MathWorks, Inc., "Fuzzy Logic Toolbox 2.2.4", http://www.mathworks.com/products/matlab/, (Septiembre de 2006)

[10] Cristiano Jacques Miosso, Adolfo Bauchspiess, (Brasil, Abril de 2001) "Fuzzy Inference System Applied to Edge Detection in Digital Images", V Brazilian Conference on Neural Networks.

[11] Jerry M.Mendel, (2000) "Uncertain Rule-Based Fuzzy Logic Systems", Prentice Hall.

[12] Juan Ramón Castro Rodríguez, (Tijuana México, Diciembre de 2005), Tesis de Maestría: "Desarrollo de Arquitecturas Híbridas Inteligentes para pronóstico de series de tiempo “, Instituto Tecnológico de Tijuana”

[13] Juan Ramón Castro Rodríguez, (La Habana Cuba, Mayo del 2000). "Desarrollo de Arquitecturas Híbridas Inteligentes para pronóstico de series de tiempo empleando Redes Neuronales, Lógica Difusa y Algoritmos Genéticos”, Congreso Internacional de Nuevas Tecnologías y Aplicaciones Informáticas

[14] Marv Pulson \& Imagedancer, (Agosto, 2006) "Digital Image Histogram Basics", Available:

http://imagedancer.com/digital_image histogram_basics.htm

Olivia Mendoza is a student of the Ph.D. program in Computer Science of the Autonomous University of Baja California. Her research interests are in neural networks, fuzzy logic and pattern recognition. She has published 5 papers in conference proceedings, edited books, and journals.

Patricia Melin is a Professor of Computer Science in the Graduate Division, Tijuana Institute of Technology, Tijuana, Mexico. In addition, she is serving as Director of Graduate Studies in Computer Science and head of the research group on fuzzy logic and neural networks. Currently, she is Vice President of HAFSA (Hispanic American Fuzzy Systems Association) and Program Chair of International Conference FNG'05. Prof. Melin is also Chair of the Mexican Chapter of the Computational Intelligence Society (IEEE). She is also Program Chair of the IFSA 2007 World Congress to be held in Cancun, Mexico. She also belongs to the Committee of Women in Computational Intelligence of the IEEE and to the New York Academy of Sciences. Her research interests are in Type-2 Fuzzy Logic, Modular Neural Networks,
Pattern Recognition, Fuzzy Control, Neuro-Fuzzy and Genetic-Fuzzy hybrid approaches. She has published over 50 journal papers, 5 authored books, 8 edited books, and 140 papers in conference proceedings.

Guillermo Licea Sandoval. Bachelor of Science in Computer Science from University of Baja California in Ensenada, a Master of Science in Computer Science from Tijuana's Institute of Technology, and a Ph. D. in Computer Science from Scientific Research and Higher Education Center of Ensenada (CICESE). Professor at University of Baja California in Tijuana since 1991. His research interests are: Computer Supported Cooperative Work, Software Engineering Tools, and Mobile Application Development. 